\title{
Multi-Person Pose Recognition Using a Zigbee Sensor Network
}

\author{
Kai-Tai Song and Chun-Wei Chen \\ Department of Electrical and Control Engineering, National Chiao Tung University \\ Hsinchu 300,Taiwan,R.O.C.(email:ktsong@mail.nctu.edu.tw; bill.ece94@nctu.edu.tw)
}

\begin{abstract}
This paper presents a novel design of a robotic multi-person posture recognition system. The proposed system consists of a human posture detection module, a wireless sensor network and a multi-person activity monitoring software running on a mobile robot. The human posture detection is based on a triaxial accelerometer and the developed posture recognition algorithms. Further, the posture detection module has a Zigbee chip and an 8-bit microcontroller on board. Thus, several posture detection modules and a Zigbee node connected to the robot control computer form a Zigbee wireless network. In the Zigbee sensor network, each posture detection module can communicate with the robot onboard computer. The multi-person activity monitoring software can monitor and record the postures of multiple users on-line in real time. A posture classification algorithm is proposed by combining timedomain analysis and wavelet transform analysis. The complete algorithm has been realized in the microcontroller of the human posture detection module. In the current design, the system can classify seven human postures: falling, standing, sitting, lying, walking, downstairs. After testing the system by five users, we have demonstrated an accurate rate of $88 \%$..
\end{abstract}

\section{INTRODUCTION}

In recent years, fast increasing of elderly population has gained much attention in our society and raised many home care discussions. In the future, more elderly people will live alone due to not enough of young people. In such circumstance, serious problems will occur if the elder people fall or got injured at home without timely help. Many studies have been conducted to develop some kinds of automatic systems to help the elderly at home (Hwang, 2004; Makikawa \& Murakami, 1996; Najafi, 2003; Sekine \& Tamura, 1998; Tanaka, 2004; Williams, 1998). These systems mostly need various types of sensors to monitor the activities of the elders. One solution is to monitor people's activity through cameras. However, in general, people do not like to be watched for a long period of time. Static cameras will not be welcome at a home setting. Other alternative approaches for such kind of applications need to be studied. In this paper, wearable activity-monitoring sensors for elders are investigated. Accelerometers are adopted to measure the acceleration of human body and the relationship between acceleration and human posture are studied.

Several posture recognition system based on inertial sensors have been reported. Bouten et al. (1997) used a triaxial accelerometer to estimate the energy consumption of human body. The system can distinguish dynamic postures with different energy consumption, but their method can not identify static postures. However, the simple relationship between energy consumption of body movement and acceleration will be valuable for classifying static and dynamic postures. Sekine and Tamura (2002) used wavelet transform to analyze the acceleration data from a triaxial accelerometer. The data of the triaxial accelerometer was separated into several frequency bands by wavelet transform.
They found Wavelet-based fractal analysis can distinguish walking, going upstairs and going downstairs human postures. But the algorithm is rather complicated and their results do not achieve real-time analysis yet.

Karantonis et al. (2006) present an implementation of a realtime classification system for the types of human movement using a single triaxial accelerometer module worn at the hip. The system can distinguish between activity and rest, measure the tilt angle of the body, and detect walking and falling conditions. They proposed an algorithm combining time domain and frequency domain and implemented the algorithm in the microcontroller. Zigbee modules were used as the signal transmission media. However, their system did not take advantage of Zigbee wireless sensor network to monitor more than one user.

It is observed that inertial sensors based human posture recognition system can be improved in three ways:

It would be better to deploy the posture recognition system in daily life environment to avoid inconvenience for users to equip with.

The posture detection system needs to achieve real-time monitoring for practical applications.

In many applications, there might be more than one person to be monitored, so the posture recognition system needs to be extended to a multi-person environment.

This paper presents a multi-person pose recognition system to meet these three conditions above. We developed a human posture estimation module using a triaxial accelerometer, a microcontroller and a Zigbee chip. The module can be wore in the belt, analyzing accelerometer data on-line in real-time and transmit the analysed results through the Zigbee chip. If 
there are several users wearing such a module, these modules will form a Zigbee wireless network. Each module is a node in the network and every node can communicate with the Zigbee receiver(coordinator) on the computer side and achieve multi-person posture monitoring

\section{THE PROPOSED SYSTEM}

The concept of the proposed multi-posture recognition system is shown in Fig. 1. The system consists of a mobile robot, a Zigbee receiver and several human posture recognition modules wore on several elderly people. The robot serves as the manger of the sensor network to monitor postures of all users in the network.

\subsection{Zigbee Sensor Network}

Receiver node: A Chipcon CC2420DBK board was adopted as the receiver of the sensor network. The board can communicate with the robot through RS-232 serial link and serves as a bridge between Zigbee network and robot onboard computer.

Human posture detection node: The human posture recognition module (see below) was designed for multiperson activity monitoring. Each module can communicate with the receiver. Several human posture recognition modules form a Zigbee sensor network including the receiver The robot can monitor several persons in this design.

\subsection{Wearable Human Posture Recognition Module}

The developed human posture recognition module is shown Fig. 2. It consists of a Freescale MMA7260QT triaxial accelerometer, an Atmel Atmega128L 8 bit Microcontroller and a Chipcon CC2420 Zigbee chip. Fig. 3 illustrates the module wore on the belt of a person.

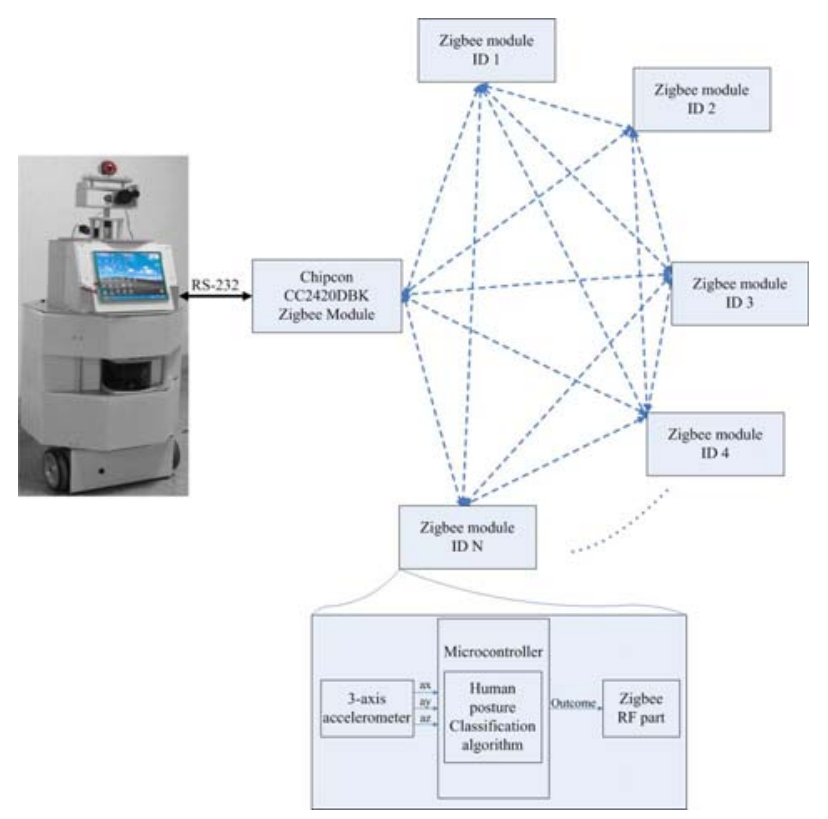

Fig. 1. Zigbee sensor network

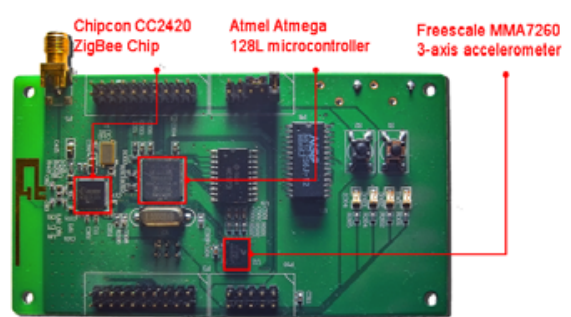

Fig. 2. Human pose estimation module

Microcontroller: An Atmega128L microcontroller serves as the computing core of the human posture recognition module. Atmega128L has seven A/D converter and eight external interrupt IO pins. There are $128 \mathrm{KBytes}$ reprogrammable flash for us to develop our algorithm. The posture classification algorithms are stored in flash ROM and running in the MCU.

Zigbee Chip: A Zigbee chip from Chipcon was adopted in this design. CC2420 is an IEEE 802.15.4 compliant chip for low-power and low-voltage wireless communication applications (Chipcon, 2004). It operates in the 2.4-GHz ISM band with an effective data rate of $250 \mathrm{~Kb} / \mathrm{s}$.

The coordinator side also has the same Zigbee chip. The human posture recognition modules can communicate with the receiver, and these modules can also communicate with each other through a mesh network. Several Zigbee modules can transmit data one by one to extent the communication range.

Accelerometer: MMA7260QT triaxial accelerometer is a capacitive-type accelerometer with four selectable sensing range: $1.5 \mathrm{~g}, 2 \mathrm{~g}, 4 \mathrm{~g}$ and $6 \mathrm{~g}$ by adjusting two pins on the chip.

\section{POSTURE RECOGNITION ALGORITHM}

The proposed recognition algorithm can classify seven human postures: falling, standing, sitting, lying, walking, going upstairs and going downstairs. As shown in Fig. 3, the recognition results are computed on line using the signals from the triaxial accelerometer on the human pose detection module. The proposed posture recognition algorithm combines time-domain analysis with wavelet transform. The complete algorithm has been implemented in the microcontroller. As shown in Fig. 4, we divide seven postures into three groups: sudden condition, dynamic postures and static postures. We first process the original acceleration signals to separate the static acceleration from the dynamic acceleration. The static acceleration comes from gravity and dynamic acceleration is produced by human motion. The dynamic acceleration is used to classify human motion-related postures, and the static acceleration is used to calculation the tilt angle of the human body. Through the measured information, we first check whether the dynamic acceleration signal is a fall condition. If the user does not fall, then we judge the user in a dynamic or static posture. If the user is static, we can classify standing, sitting and lying from the angle of the user torso. If the user is in dynamic motion, we detect whether there are paces or not. If paces are detected, the acceleration signal will be analyzed by wavelet transform 


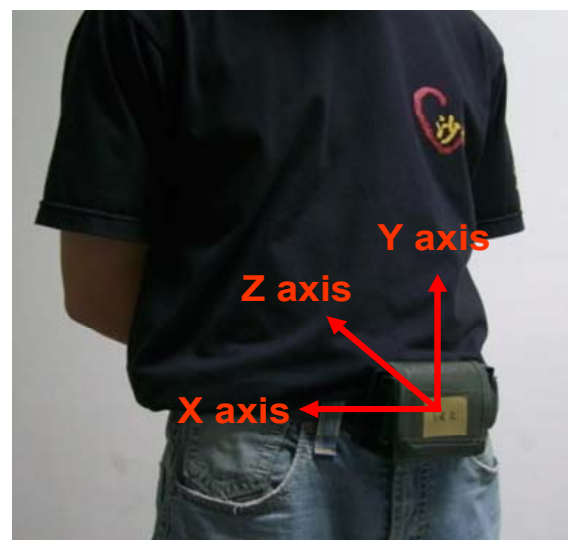

Fig. 3. Sensor axis of the human posture detection

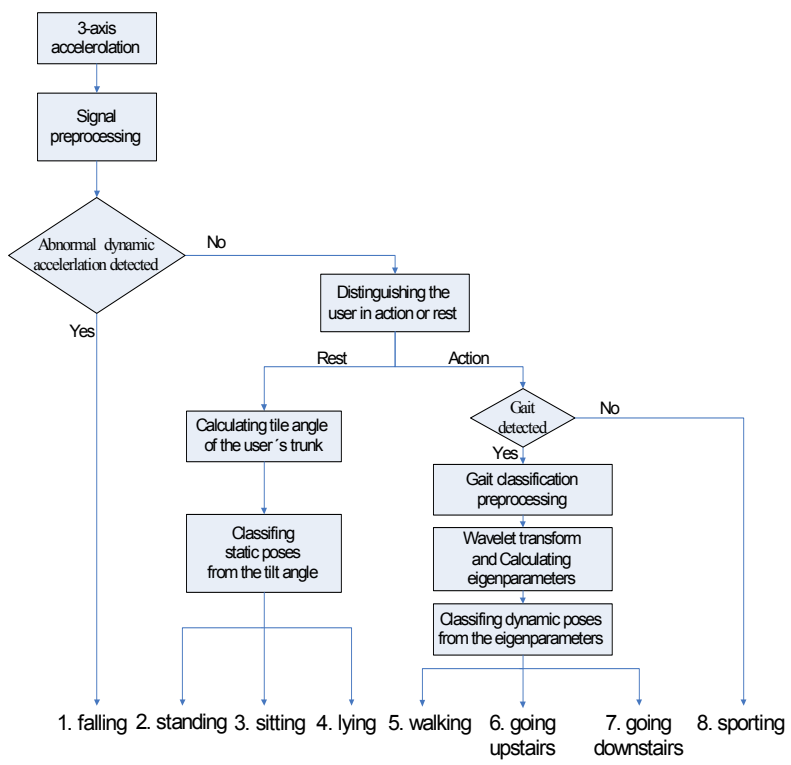

Fig. 4. Human posture estimation algorithm

to identify the paces from walking, going upstairs or going downstairs. If no paces are detected, the result will be sporting.

\subsection{Signal Pre-processing}

In this design, a sampling frequency of $128 \mathrm{~Hz}$ was selected for acceleration data acquisition. In order to reduce the system response time and collect enough data to analyze the human posture, we estimate human posture once every two seconds. Thus, 256 samples of three-direction acceleration are acquired every two seconds. We apply eight layers of Haar wavelet transform to the 256 samples. The eighth layer coefficients represent the frequency band of $0 \sim 0.25 \mathrm{~Hz}$. We then apply inverse wavelet transform to the eighth layer coefficient to represent the static acceleration in the two seconds period. The dynamic acceleration can be obtained by subtracting the static acceleration from the original signal. Fig. 5 shows the original signal of walking and the separated static and dynamic accelerations from the original signal. The static acceleration comes from the Earth's gravity.

\subsection{Moving and Rest}

Signal magnitude area (SMA) of dynamic acceleration is highly correlated with energy of movement (Karantonis et al., 2006). One can identify dynamic and static posture through SMA by setting a proper threshold.

$$
S M A=\sum_{n=0}^{256}\left(\left|a_{x_{-} \text {dynamic }}[n]\right|+\left|a_{y_{-} \text {dynamic }}[n]\right|+\left|a_{z_{-} \text {dynamic }}[n]\right|\right)
$$

Fig. 6 shows an SMA result of three static postures, namely standing, sitting and lying. The SMA of static postures is small, while those of three dynamic postures, namely walking, going upstairs and going downstairs are much lager. So we take SMA threshold $=50 \mathrm{~g}$ to distinguish dynamic and static postures.

\subsection{Fall Detection}

Karantonis et al. (2006) proposed a parameter termed signal vector magnitude (SMV) to estimate the condition of falling:

$$
S M V=a_{x_{-} \text {dynamic }}^{2}+a_{y_{-} \text {dynamic }}^{2}+a_{z_{-} \text {dynamic }}^{2}
$$

Because we estimate human postures every two seconds,
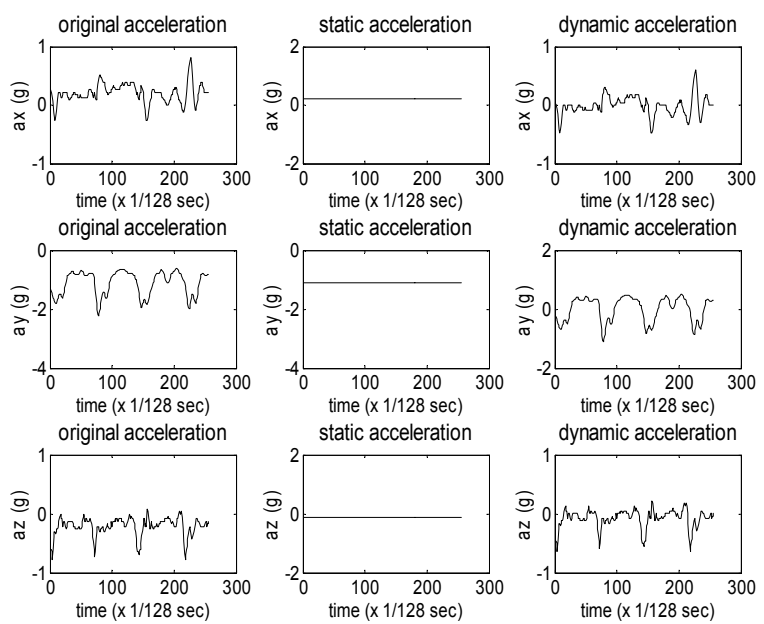

Fig. 5. Pre-processing of walking signals

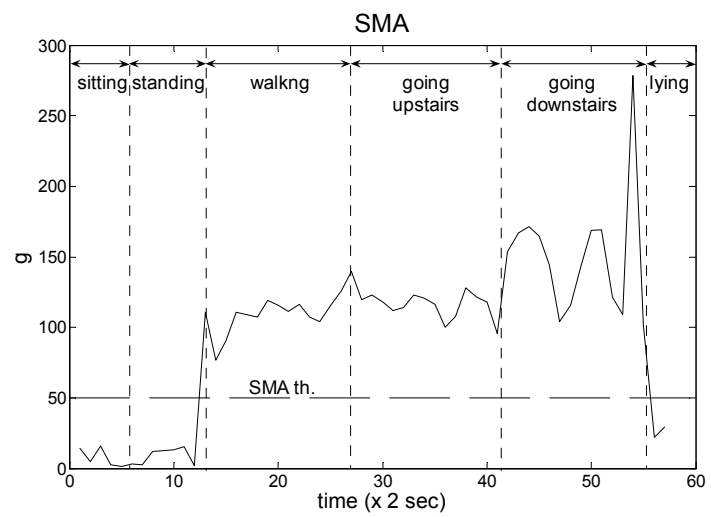

Fig. 6. Dynamic and static postures classified by SMA 


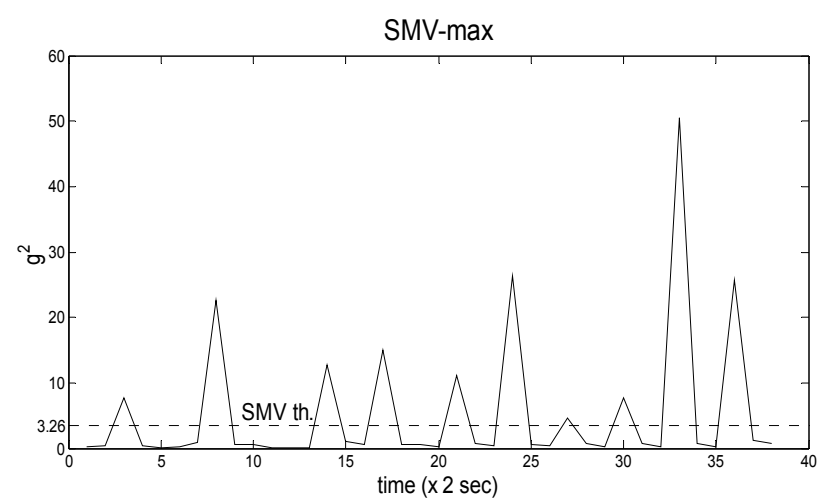

Fig. 7. Detecting falling by SMV_max

there will be $256 \mathrm{SMV}$ values. We choose the largest SMV in two seconds and define it as SMV_max to estimate falling. Fig. 7 shows a test result of a user in a room without any constrain and fell down ten times during a test. The ten peaks in the figure are the event of falling. As shown in the figure, one can estimate whether a user falls by checking SMV_max $>3.24$.

\subsection{Standing, Sitting and Lying}

Three static postures, namely standing, sitting and lying can be recognized by measuring the tilt angle of the body torso. The torso tilt angle can be measured via three-direction static acceleration. If human torso tilted more than 60 degrees extent, the state of lie down can be determined. A tilt angle between 20 to 60 degrees is most likely the state of sit down, while a tilt angle of 0 to 20 degrees is the state of standing. Fig. 8 illustrates the relationship of $y$ - and z-direction static accelerations caused by Earth gravity and body posture sensing.

\subsection{Walking, Going Upstairs and Going Downstairs}

The human motion of walking, going upstairs and going downstairs are analyzed and determined through wavelet transform. However, for wavelet transform one would need more data samples in order to obtain reliable results. In this

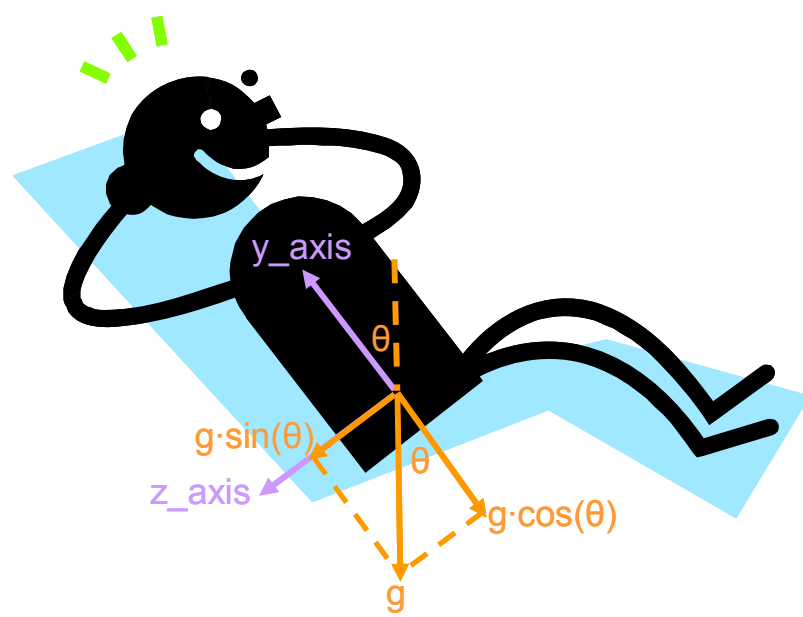

Fig. 8. Relationship of static acceleration and tilt angle
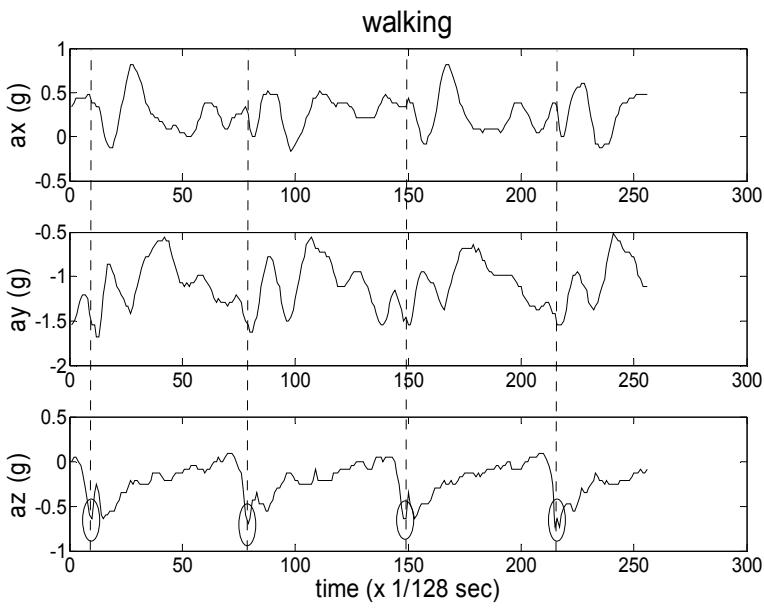

Fig. 9. Acceleration of walking
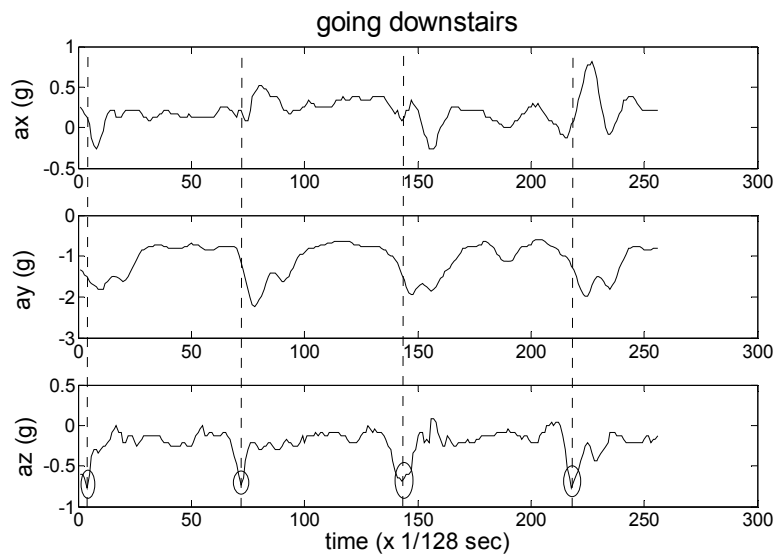

Fig. 10. Acceleration of going downstairs
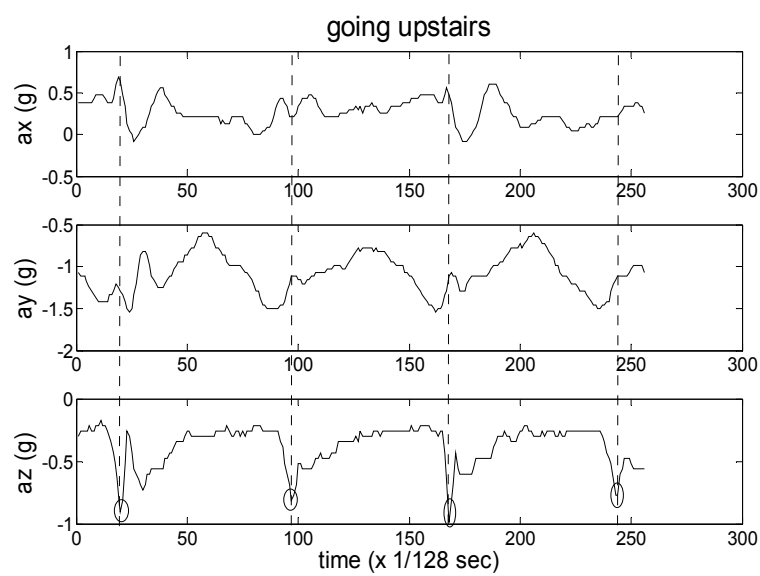

Fig. 11. Acceleration of going upstairs

design, our approach is to increase data for recognition processing, but do not increase the sampled data in order not to make the response time longer. For instance, Figs. 9 11 show the acceleration waveform of walking, going upstairs and going downstairs respectively. It can be observed that the acceleration waveforms of the three motion types are periodic We can identify the start-up of each step by the local minimum of $\mathrm{z}$-axis acceleration. The acceleration values 
between two successive local minimum represent acceleration signal of one step.

Due to different foot-step strength of left and right leg, we capture consecutive two paces to obtain a reliable analysis. Consequently, we extend the data acquisition interval to 512 samples to enrich the data for wavelet transform. We apply the extended 512 data for Haar wavelet transform to separate the signals into various frequency bands. The frequency band of human walking is $1 \sim 16 \mathrm{~Hz}$. We use the wavelet coefficients of the frequency band to classify the three walking pattern.

Further, it is observed that among the three walking patterns, the wavelet coefficients of going upstairs is the smallest in the $\mathrm{z}$-axis direction. But the total power of the three walking patterns is almost the same. So we define a parameter called the ratio of total power to signal in front direction (RTF) to identify the posture of going upstairs such that:

$$
R T F=\frac{\left\|a_{z}\right\|_{2}^{2}}{\sum_{j=3}^{6} d_{z j}}
$$

As for classification of going downstairs and walking, acceleration of going downstairs is larger in y-axis but smaller in z-axis. We define another parameter called the ratio of vertical power to frontal power (RVF) to identify going downstairs such that:

$$
R V F=\frac{\sum_{j=3}^{6} d_{y j}}{\sum_{j=3}^{6} d_{z j}}
$$

Through these two parameters one can classify walking, going upstairs and going downstairs. Fig. 12 shows the

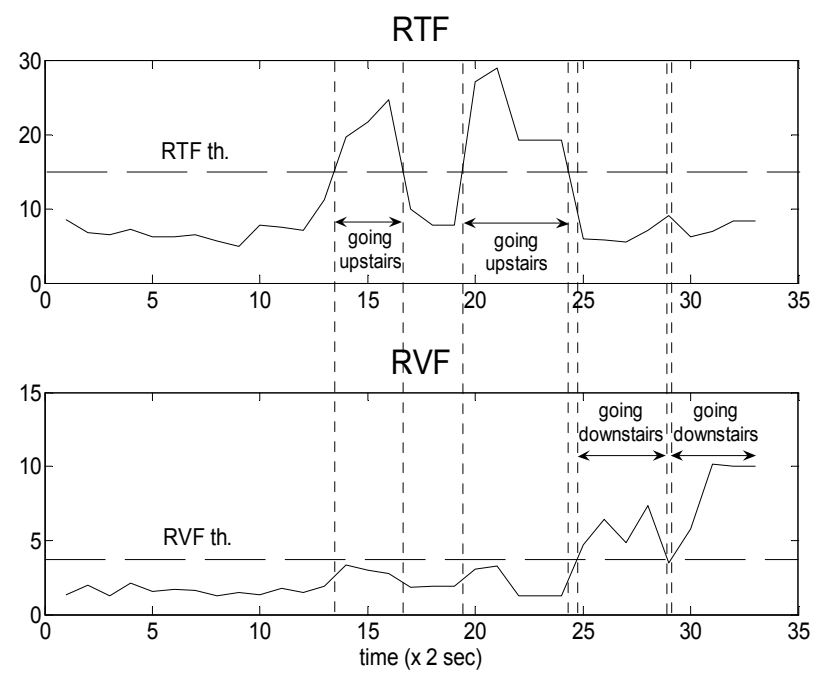

Fig. 12 Classify walking, going upstairs and going downstairs by RTF and RVF distribution of the two parameters in the three walking patterns.

\section{EXPERIMENTAL RESULTS}

In order to evaluate the proposed algorithms and the developed posture recognition system, we designed three experiments to test the recognition accurate rate of the seven postures: 1.sitting, 2. standing, 3. walking, 4. going upstairs, 5. going downstairs, 6 . fall and 7. lying.

\subsection{Single tester, Individual Posture recognition}

In this experiment, five individual testers wore the posture recognition module and exercised the seven postures. Each of the five testers demonstrated each posture twenty times and the recognition results are recorded for the seven postures. The experimental results are shown in Table1. It can be seen that the recognition rates are all above $85 \%$ except for the posture of going down stares.

\subsection{Single Tester, Continuous Posture Recognition}

In order to evaluate the functionality of posture recognition as well as the monitoring software, five testers exercised seven postures continuously in this experiment. Each tester demonstrated seven postures continuously for twenty times and the recognition results were recorded. The experimental results are shown in Table 2 . We see that the recognition rates are similar to the previous results of separate posture demonstration.

\subsection{Multi-Tester, Continuous Posture Recognition}

In this experiment, three testers demonstrated certain posture individually and concurrently. The recognition rates of the demonstrated postures were detected in real time and recorded by the human posture monitoring system. Two groups of three testers participated the experiment. The

Table 1. Experimental results of individual posture recognition

\begin{tabular}{|l|c|c|c|c|c|c|c|}
\hline $\begin{array}{c}\text { Actual } \\
\text { posture } \\
\text { mati- } \\
\text { posture }\end{array}$ & 1 & 2 & 3 & 4 & 5 & 6 & 7 \\
\hline 1.Sitting & 89 & 0 & 0 & 0 & 0 & 0 & 0 \\
\hline 2.Standing & 11 & 100 & 0 & 0 & 0 & 0 & 0 \\
\hline 3.Walking & 0 & 0 & 91 & 10 & 14 & 0 & 0 \\
\hline 4.Upstairs & 0 & 0 & 5 & 85 & 3 & 0 & 0 \\
\hline 5.Downstai & 0 & 0 & 4 & 0 & 79 & 0 & 0 \\
\hline 6.Fall & 0 & 0 & 0 & 0 & 1 & 94 & 0 \\
\hline 7.Lying & 0 & 0 & 0 & 0 & 0 & 0 & 100 \\
\hline 8. Transit & 0 & 0 & 0 & 5 & 3 & 6 & 0 \\
\hline $\begin{array}{l}\text { Recog-nition } \\
\text { rate }\end{array}$ & 89 & 100 & 91 & 85 & 79 & 94 & $\begin{array}{c}100 \\
\% \\
\%\end{array}$ \\
\hline
\end{tabular}


Table 2. Experimental results of single tester continuous posture recognition

\begin{tabular}{|l|c|c|c|c|c|c|c|}
\hline $\begin{array}{c}\text { Actual } \\
\text { posture } \\
\begin{array}{l}\text { Esti- } \\
\text { mated } \\
\text { Posture }\end{array}\end{array}$ & 1 & 2 & 3 & 4 & 5 & 6 & 7 \\
\hline 1.Sitting & 20 & 0 & 0 & 0 & 0 & 0 & 0 \\
\hline 2.Standing & 1 & 19 & 3 & 0 & 0 & 0 & 0 \\
\hline 3.Walking & 0 & 0 & 72 & 1 & 7 & 0 & 0 \\
\hline 4.Upstairs & 0 & 0 & 1 & 28 & 1 & 0 & 0 \\
\hline 5.Downstai & 0 & 0 & 3 & 0 & 27 & 0 & 0 \\
\hline 6.Fall & 0 & 0 & 0 & 0 & 1 & 5 & 0 \\
\hline 7.Lying & 0 & 0 & 0 & 0 & 0 & 0 & 14 \\
\hline 8.Transit & 0 & 0 & 0 & 6 & 0 & 0 & 0 \\
\hline $\begin{array}{l}\text { Recog- } \\
\text { nition rate }\end{array}$ & 95 & 100 & 87 & 80 & $\begin{array}{c}75 \\
\%\end{array}$ & $\begin{array}{c}100 \\
\%\end{array}$ & $\begin{array}{c}100 \\
\%\end{array}$ \\
\hline
\end{tabular}

Table 3. Experimental results of multi-person posture recognition

\begin{tabular}{|l|c|c|c|c|c|c|c|}
\hline $\begin{array}{r}\text { Actual } \\
\text { posture }\end{array}$ & 1 & 2 & 3 & 4 & 5 & 6 & 7 \\
$\begin{array}{l}\text { Esti- } \\
\text { mated } \\
\text { Posture }\end{array}$ & 25 & 0 & 0 & 0 & 0 & 0 & 0 \\
\hline 1.Sitting & 2 & 26 & 0 & 0 & 0 & 0 & 0 \\
\hline 2.Standing & 0 & 0 & 62 & 2 & 4 & 0 & 0 \\
\hline 3.Walking & 0 & 0 & 4 & 40 & 2 & 0 & 0 \\
\hline 4.Upstairs & 0 & 0 & 4 & 3 & 37 & 1 & 0 \\
\hline 5.Downstai & 0 & 0 & 0 & 0 & 0 & 5 & 0 \\
\hline 6.Fall & 0 & 0 & 0 & 0 & 0 & 0 & 26 \\
\hline 7.Lying & 0 & 0 & 2 & 3 & 3 & 0 & 0 \\
\hline 8. Transit & 93 & 100 & 91 & 83 & 80 & 83 & 100 \\
\hline$\%$ & $\%$ & $\%$ & $\%$ & $\%$ & $\%$ \\
\hline $\begin{array}{l}\text { Recog- } \\
\text { nition rate }\end{array}$ & $\%$ & $\%$ & $\%$ & & \\
\hline
\end{tabular}

experimental results are presented in Table 3.

\section{CONCLUSION}

This paper presents a real-time human posture recognition system based on accelerometers. The proposed recognition algorithm classifies seven human postures: fall, standing, sitting, lying, walking, going upstairs and going downstairs. The developed posture recognition module contains a Zigbee chip and several human posture estimation modules can form a Zigbee network to monitor several people at the same time. After testing the system for five users, a total recognition rate of $88 \%$ has been obtained. In the future, the posture recognition system will be integrated with a mobile robot for investigating various home-care applications.

\section{ACKNOWLEDGMENT}

This work was partly supported by the National Science Council, Taiwan, R.O.C, under grant NSC-95-2218-E-009007 and the Ministry of Economic Affairs under grant 95EC-17-A-04-S1-054.

\section{REFERENCES}

Bouten, C. V. C., Koekkoek, K. T. M., Verduin, M., Kodde, R. and Janssen, J. D. (1997). A triaxial accelerometer and portable data processing unit for the assessment of daily physical activity, IEEE Trans. Biomedical Engineering, vol.44, no.3, pp. 136-147.

Chipcon, Inc. (2004). CC2420 $2.4 \quad \mathrm{GHz}$ IEEE 802.15.4/Zigbee-ready RF transceiver datasheet.

Hwang, J. Y., Kang, J.M., Jang, Y. W. and Kim, H. C. (2004). Development of novel algorithm and real-time monitoring ambulatory system using Bluetooth module for fall detection in the elderly, Proceeding of the 26th Annual International Conference of the IEEE EMBS, San Francisco, USA, pp. 2204-2207.

Karantonis, D. M., Narayanan, M. R., Mathie, M., Lovell, N. H. and Celler, B. G.(2006). Implementation of a realtime human movement classifier using a triaxial accelerometer for ambulatory monitoring, IEEE Trans. Information Technology in Biomedicine, vol. 10, no. 1, pp. 156-167, 2006

Makikawa, M. and Murakami, D. (1996). Development of an ambulatory physical activity and behavior map monitoring system, Proceeding of the 18th Annual International conf. of the IEEE engineering in medicine and biology society, Amsterdam, Netherlands, pp.71-72.

Najafi, Aminian, B., K., Parachiv-Ionescu, A., Loew, F. and Robert, P. (2003). Ambulatory system for human motion analysis using a kinematic sensor: monitoring of daily physical activity in the elderly. IEEE Trans. Biomedical Engineering, vol.50, issue 6, pp.711-723.

Sekine, M. and Tamura, T. (1998). Classification of acceleration waveform in a continuous walking record, Proceeding of the 20th Annual International Conf. Medicine and Biology Society, Hong Kong, China, pp. 1523-1526.

Sekine, M. and Tamura, T.(2002). Discrimination of walking pattern using wavelet-based fractional analysis, IEEE Trans. Neural Systems and Rehabilitation Engineering, vol.10, issue 3, pp. $188-196$

Tanaka, S., Motoi, K., Nogawa, M. and Yamakoshi, K. (2004). A new portable device for ambulatory monitoring of human posture and walking velocity using miniature accelerometers and gyroscope, Proceedings of the 26th Annual International Conference of the IEEE EMBS, San Francisco, USA, pp. 2283-2286.

Williams, G., Doughty, K., Cameron, K. and Bradly, D. A. (1998). A smart fall and activity monitor for telecare application, Proceedings of the 20th Annual International Conf. of the IEEE engineering in medicine and biology society, Hong Kong, China, pp.1151-1154. 